American Journal of Applied Sciences 5 (6): 618-625, 2008

ISSN 1546-9239

(C) 2008 Science Publications

\title{
Using IRS Products to Recover 7ETM+ Defective Images
}

\author{
${ }^{1}$ Mobasheri Mohammad Reza and ${ }^{2}$ Sadeghi Naeini Ali \\ ${ }^{1}$ Department of Remote Sensing Engineering, K. N. Toosi University of Technology, \\ P.O. Box 15875-4416 Tehran, Iran \\ ${ }^{2}$ Iran Space Agency, Tehran, Iran
}

\begin{abstract}
On May 31st, 2003, Landsat 7 faced an anomaly in the Scan Line Corrector (SLC) normal operation. This malfunctioning of SLC caused the individual scan lines alternately overlap each other and consequently produce large gaps at the edges of the image. Regarding the unique specification of $\mathrm{ETM}^{+}$sensor on board of Landsat-7 satellite such as its spectral bands in the shortwave IR and TIR region and its suitable spatial resolution which is ideal for most of the scientific researches, a technique for the reconstruction of the defected images due to the SLC malfunctioning, was built up. Due to the availability of IRS/1D LISS-III images for our region of interest i.e., southwest of Iran, it was decided to use these images to recover $7 \mathrm{ETM}^{+}$products. The procedure in reconstructing the defected $7 \mathrm{ETM}^{+}$ images is divided in to two stages. In the first stage, after implementation of some preprocessing to both LISS-III and 7ETM ${ }^{+}$, a linear regression model between bands 3 and 4 of $7 \mathrm{ETM}^{+}$and bands 2 and 3 of LISS-III was setup. This model is used to fill up the missing places in $7 \mathrm{ETM}^{+}$defected image and produced two new images in bands 3 and 4 . In the second stage, these two newly reconstructed images of $7 \mathrm{ETM}^{+}$were used to recover images of $7 \mathrm{ETM}^{+}$in other spectral bands. At this stage, two methods were introduced, one using linear relationship between band 3 and bands 1 and 2 and in the second method we introduced a planar relationship between bands 3 and 4 with each one of bands 5, 6 and 7 . The models are applied to few images and are found to be fairly reliable. The primary and necessary conditions for applying these methods have been explained in detail.
\end{abstract}

Keywords: Remote sensing, 7ETM ${ }^{+}$, SLC, IRS/1D-LISSIII, image reconstruction

\section{INTRODUCTION}

Landsat-7 satellite which nowadays is the latest version of the Landsat series, was launched in April 15th, 1999 after unsuccessful launching of Landsat-6 in May 10th, 1993. Before launching Landsat-7, only Landsat 4 and 5 satellites were providing service to the remote sensing users all over the world. Landsat-7 bore the $7 \mathrm{ETM}^{+}$sensor which was much more advanced than the TM sensor. The two main differences between $7 \mathrm{ETM}^{+}$and TM sensors besides the advancement of the technology are first in the addition of a panchromatic band with a spatial resolution of $15 \mathrm{~m}$ in a $0.5-0.9 \mathrm{~m}$ region of the electromagnetic spectrum and secondly in improving the spatial resolution of the thermal band 6 from 120 to $60 \mathrm{~m}^{[1,5]}$. Information on the Landsat satellites as well as the spectral characteristics of their sensors has been provided in Table 1 .
On May 31st, 2003, Landsat 7 faced an anomaly in the Scan Line Corrector (SLC) normal operation ${ }^{[2,4]}$. This malfunctioning of SLC caused individual scan lines alternately overlap each other and consequently produce large gaps at the edges of the image. Many attempts have so far been devoted to find a way to compensate for the missing data ${ }^{[3,8]}$. While it is not quite possible to correct for these missing data, it might be possible to modify the processing procedures to produce images containing enough acceptable pixels for some applications. Among these applications are Geography, Agriculture, Forestry, Rangeland, Ecosystems, Glaciology and Ice Cap Monitoring, Ecological Remote Sensing, Phenological Characterization, Coastal/Oceanographic Remote Sensing and Corals. Among these applications are Geography, Agriculture, Forestry, Rangeland, Ecosystems, Glaciology and Ice Cap Monitoring, Ecological Remote Sensing, Phenological

Corresponding Author: Mohammad Reza Mobasheri, Department of Remote Sensing Engineering, K. N. Toosi University of Technology, P.O. Box 15875-4416 Tehran, Iran Tel: +98 9121226630 Fax: +98 2188786213 
Am. J. Applied Sci., 5 (6): 618-625, 2008

\begin{tabular}{|c|c|c|c|c|}
\hline Satellite & $\begin{array}{l}\text { Revisit } \\
\text { time } \\
\text { (days) }\end{array}$ & Sensor & $\begin{array}{l}\text { Bandwidth } \\
\text { (microns) }\end{array}$ & $\begin{array}{l}\text { Resolution } \\
\text { (m) }\end{array}$ \\
\hline \multirow[t]{7}{*}{ Landsat-1, 2, 3} & 18 & RBV & (1) $0.48-0.57$ & 80 \\
\hline & & & (2) $0.58-0.68$ & 80 \\
\hline & & & (3) $0.70-0.83$ & 80 \\
\hline & & MSS & (4) $0.5-0.6$ & 79 \\
\hline & & & (5) $0.67-0.7$ & 79 \\
\hline & & & (6) $0.7-0.8$ & 79 \\
\hline & & & (7) $0.8-1.1$ & 79 \\
\hline \multirow[t]{11}{*}{ Landsat- 4 and 5} & 16 & MSS & (4) $0.5-0.6$ & 82 \\
\hline & & & (5) $0.67-0.7$ & 82 \\
\hline & & & (6) $0.7-0.8$ & 82 \\
\hline & & & (7) $0.8-1.1$ & 82 \\
\hline & & $\mathrm{TM}$ & (1) $0.45-0.52$ & 30 \\
\hline & & & (2) $0.52-0.60$ & 30 \\
\hline & & & (3) $0.63-0.69$ & 30 \\
\hline & & & (4) $0.76-0.90$ & 30 \\
\hline & & & (5) $1.55-1.75$ & 30 \\
\hline & & & (6) $10.4-12.5$ & 120 \\
\hline & & & (7) $2.08-2.35$ & 30 \\
\hline \multirow[t]{8}{*}{ Landsat-7 } & 16 & $7 \mathrm{ETM}^{+}$ & (1) $0.45-0.52$ & 30 \\
\hline & & & (2) $0.52-0.60$ & 30 \\
\hline & & & (3) $0.63-0.69$ & 30 \\
\hline & & & (4) $0.76-0.90$ & 30 \\
\hline & & & (5) $1.55-1.75$ & 30 \\
\hline & & & (6) $10.4-12.5$ & 60 \\
\hline & & & (7) $2.08-2.35$ & 30 \\
\hline & & & (pan) $0.5-0.9$ & 15 \\
\hline
\end{tabular}

Characterization, Coastal/Oceanographic Remote Sensing and Coral Reef Monitoring, Tropical Forest Monitoring, Water Quality Monitoring, Remote Sensing Methodology and Techniques Development, and Global Change Monitoring. Initial evaluation shows that the processed defected images maintained radiometric and geometric fidelity to an acceptable level. Also EDC scientists and engineers performed evaluations of the radiometric and geometric validity of these products ${ }^{[3,5]}$.

Many others used basic interpolation algorithms, to fill in some of the missing pixels.

However, the data produced with interpolation techniques requires further research and may not be useful for all scientific applications ${ }^{[4]}$. For other applications such as precision farming however, the presence of the anomaly and associated missing pixels does degrade the usefulness of the imagery.

We believe that the anomalous Landsat 7 data products may retain significant and important utility for scientific applications if we use images of some other operational sensors on board of other platforms to fill up the gaps ${ }^{[6]}$.

In this regards, the potential to develop new tools or methods of compensation for this anomaly, like developing a mosaic of overlapping scenes or data from other sensors products to fill in missing pixels, may enable even more scientific use of these data.

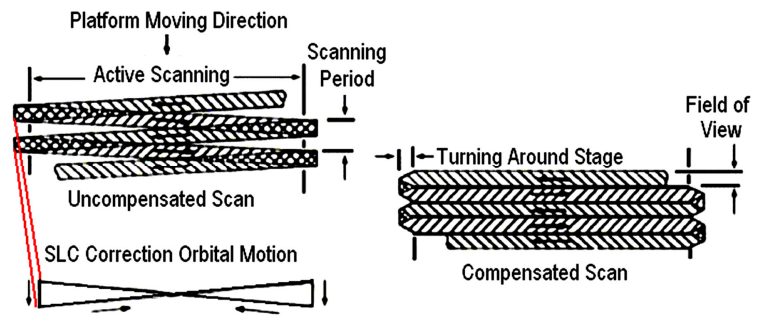

Fig. 1: Modality of scanning mirror and SLC operation on $7 \mathrm{ETM}^{+}$

The SLC is an electromechanical device that can compensates for the forward motion of the satellite during $7 \mathrm{ETM}^{+}$image scanning. It allows successive forward and backward scans of the $7 \mathrm{ETM}^{+}$scanning mirror to image in a parallel scanning series (Fig. 1).

With a defected SLC, the 7ETM ${ }^{+}$scans the scene in a pattern similar to Fig. 4.

Instead of aligning in parallel scans, the individual scans alternately overlap in the center while leaving large gaps or at the edge of the images. Then only in the center of the image (nadir looking condition) scans give full contiguous coverage of the surface scanned below the satellite.

In this study it is intended to recover the $7 \mathrm{ETM}^{+}$ defected images using other sensors on board of other platforms which we will call auxiliary images from now on. These auxiliary images must be produced in a short time after/before $7 \mathrm{ETM}^{+} \mathrm{s}$ where we believe the surface patterns in the scene have not been changed seriously. In this case, we might be able to fill the missing places in $7 \mathrm{ETM}^{+}$images properly and scientifically. The auxiliary images should have the following characteristics: a)- having spatial and radiometric resolution as close as possible to those of $7 \mathrm{ETM}^{+} \mathrm{s}$. b)being produced in spectral bands as similar as possible to those of $7 \mathrm{ETM}^{+} \mathrm{s}$ and c)- the time distance between $7 \mathrm{ETM}^{+}$and auxiliary images being as short as possible to reduce the change in the land cover as much as possible. Of course access to these data and the expenditures must be justified with the importance of the project in hand. Due to the availability of the LISSIII sensor images on board of IRS/1D in $\operatorname{Iran}^{[5]}$ and its relatively low price, these products were used as auxiliary images in this work. The first Indian Remote Sensing Satellite (IRS/1A) was launched on March 17th, 1988. Later IRS/1C and IRS/1D were launched on Dec., 1995 and Sep.,1997, respectively where we had the receiving station for these two satellites in $\operatorname{Iran}^{[5]}$.

Three sensors were on board of these satellites LISS-III, PAN and WiFS among them LISS-III products were used in this study. The characteristics of 
Table 2: Comparison between $7 \mathrm{ETM}^{+}$and LISS-III sensors characteristics

\begin{tabular}{lll}
\hline 7ETM ${ }^{+}$(Landsat) & LISS-III $(\mathrm{IRS})$ & Subject \\
\hline $0.630-0.690 \mu \mathrm{m}$ & $0.620-0.800 \mu \mathrm{m}$ & ${\text { Band } 37 \mathrm{ETM}^{+}}^{+}$ \\
$0.750-0.900 \mu \mathrm{m}$ & $0.770-0.860 \mu \mathrm{m}$ & ${\text { Band } 4 \mathrm{ETM}^{+}}^{+}$ \\
Available & Not available & Thermal band \\
98.2 & 98.69 & Inclination angle \\
$30 \mathrm{~m}$ & $23.5 \mathrm{~m}$ & Pixel dimension at \\
& & Nadir looking \\
$705 \mathrm{~km}$ & $817 \mathrm{~km}$ & Satellite altitude \\
$185 \mathrm{~km}$ & $141 \mathrm{~km}$ & Swath-width \\
$16 \mathrm{days}$ & $24 \mathrm{days}$ & Temporal resolution \\
Sunsynchronize & Sunsynchronize & Orbit \\
10 am \pm 15 min & $10: 30$ am $\pm 5 \mathrm{~min}$ & Tropical passage \\
Lcal & Local & time \\
\hline
\end{tabular}

LISS-III and $7 \mathrm{ETM}^{+}$is compared in Table 2 and we found this sensor the best for our aim.

\section{MATERIALS AND METHODS}

The first Indian Remote Sensing Satellite (IRS/1A) was launched on March 17th, 1988. Later IRS/1C and IRS/1D were launched on Dec., 1995 and Sep., 1997 respectively where we had the receiving station for these two satellites in $\operatorname{Iran}^{[5]}$. Three sensors were on board of these satellites LISS-III, PAN and WiFS among them LISS-III products were used in this study. The characteristics of LISS-III and $7 \mathrm{ETM}^{+}$is compared in Table 2 and we found this sensor the best for our aim.

Recovering the empty space in $7 \mathrm{ETM}^{+}$images using LISS-III, can be done in two stages. In the first stage, we try to build up $7 \mathrm{ETM}^{+}$image in band 3 and 4 using Bands 2 and 3 of LISS-III and in the second stage the results of $7 \mathrm{ETM}^{+}$band 3 and 4 will be used to build up bands 1, 2, 5 and 6 of $7 \mathrm{ETM}^{+}$s.

For this we need to implement few preprocesses on LISS-III as the following steps:

Stage 1: Filling up empty spaces in bands 3 and 4 of 7ETM ${ }^{+}$image Due to the fact that only bands of 3 and 4 of $7 \mathrm{ETM}^{+}$and LISS-III are comparable (Table 2), we first use LISS-III bands to fill up empty spaces of equivalent bands of 7ETM.

Step 1: We receive images of LISS-III at LEVEL-0 in $\mathrm{a} *$.irl format. The first step is to transform this file to a raster file with a format *.hdf

Step 2: The next step is radiometric corrections on *.hdf files and removing instrumental errors as well as applying the calibration coefficients to each and individual pixels of the images. This is because pixels of each line are being imaged with a particular CCD cell. The image passed the step will be called LEVEL-1B.

Step 3: At this step, geometrical corrections will be done i.e., each pixel will be assigned a set of coordinates with respect to an earth system. It is natural that parameters such as panoramic effect, sensor oblique viewing in the edges and corners of the image, spherical shape of the earth and its revolution, change in the state and location of the satellite and its relative speed, orbital parameters of the platform and etc, all will be responsible for any deterioration of the image. In geometrical correction step one can remove or reduce these effects and geo-referencing the image somehow that each pixel finds its real position on the earth surface.

Step 4: Now the pixel dimensions in the LISS-III images should be resized to those of ETMs (can be found in the header file). Note that the geometrical precision at this stage completely depends on the satellite orbital parameters and sensor attitude (tilt, yaw and pitch). In the case of LISS-III images, it is possible to shift the image about 5 kilometers (due to the lifetime of IRS orbital parameters) with respect to the ground truth. As a result, one stage precise geometrical correction using first or second order polynomials is inevitable. These GCPs can be determined using a GPS set, available maps of the region or available already geo-referenced images. Usually using an affine model for the precise geometrical correction at this stage would suffice.

Step 5: Since the reason for using LISS-III images is to recover the missing information in $7 \mathrm{ETM}^{+}$images, these two images should be completely equivalent from geometrical and geographical aspects. Based on this, the LISS-III to $7 \mathrm{ETM}^{+}$registration should be done at this stage.

Step 6: To reduce the effects of resampling on the grey level values of LISS-III, we used the nearest neighbor's sampling method. Keeping the available coordinates in header file, we tried to reduce the number of sampling on $7 \mathrm{ETM}^{+}$and only do the sampling on LISS-III.

Step 7: Due to the differences in Landsat-7 and IRS/1D orbits and Swath Width (185 and $141 \mathrm{Km}$ respectively), usually one scene of 7ETM ${ }^{+}$will be fully covered by 6 scenes of IRS/1D. In the present work, we used Row38, Path-165 of $7 \mathrm{ETM}^{+}$defected image of Jan 12th, 
Am. J. Applied Sci., 5 (6): 618-625, 2008

Table 3: Date, Path and Row of 6 IRS/1D images covering 7ETM ${ }^{+}$ image of Jan 12, 2005

\begin{tabular}{lc}
\hline Date of acquisition & Path row \\
\hline $15 / 1 / 2005$ & 66,48 \\
$15 / 1 / 2005$ & 66,49 \\
$18 / 12 / 2004$ & 67,48 \\
$18 / 12 / 2004$ & 67,49 \\
$9 / 1 / 2005$ & 68,48 \\
$9 / 1 / 2005$ & 68,49 \\
\hline
\end{tabular}

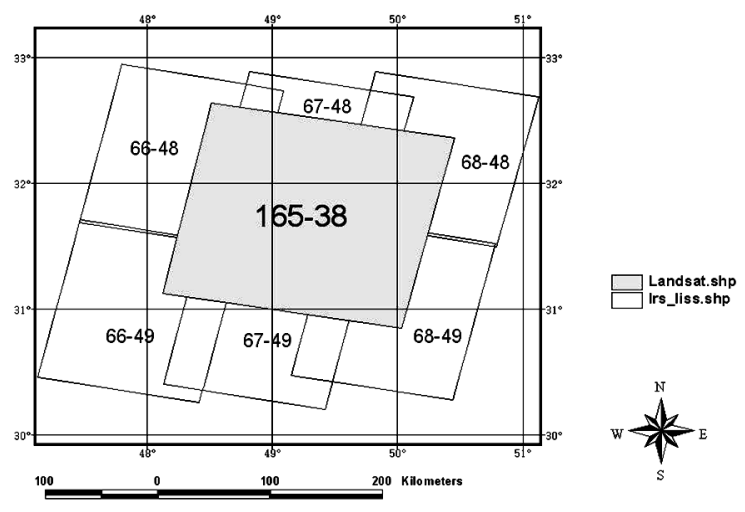

Fig. 2: Schematic diagram of 6 IRS/1D and one $7 \mathrm{ETM}^{+}$images in the region

2005. The scene was covered with 6 LISS-III images details of which can be found in Table 3 as well as Fig. 2.

Step 8: Due to the 3 to 24 days time difference between LISS-III and $7 \mathrm{ETM}^{+}$images, it is possible to have serious changes in atmospheric conditions, soil moisture and vegetation cover in the scenes. Also there might be minor differences in the spectral radiances in the red and NIR bands. Then a simple mosaic of 6 IRS images would not be appropriate because later there would be serious sources of error on radiometric differences in the missing regions of the $7 \mathrm{ETM}^{+}$.

To solve this problem, we decided to first fit a mathematic model to the similar spectral bands of these two sensors. Then using this model, we would be able to predict the missing data of $7 \mathrm{ETM}^{+}$image from IRS/1D.

Since six LISS-III images were from 3 different dates, one image from each date was selected and from the common regions of $7 \mathrm{ETM}^{+}$and LISS-III, DNs in the red and NIR band were compared and studied. About 100 samples in these regions were selected and their DNs were extracted and compared and high correlations between these two sets of DNs were found. This means that a linear model for each date would relate $7 \mathrm{ETM}^{+}$and LISS-III images in each band to an
Table 4: List of calculated coefficients $\mathrm{a}$ and $\mathrm{b}$ as in equation $\mathrm{ETM}^{+}=$ $\mathrm{a}$. LISS $+\mathrm{b}$ for Red and NIR spectral bands

\begin{tabular}{lllll}
\hline $\begin{array}{l}\text { Correlation } \\
\text { coeff. for red }\end{array}$ & A Red & B Red & Path & Date \\
\hline 0.932 & 0.7462 & 1.7493 & 66 & $15 / 1 / 2005$ \\
0.93 & 0.7007 & 2.6638 & 67 & $18 / 12 / 2005$ \\
0.97 & 1.0021 & 23.903 & 68 & $9 / 1 / 2005$ \\
$\begin{array}{l}\text { Correlation } \\
\text { Coeff. for NIR }\end{array}$ & B NIR & A NIR & Path & Date \\
\hline 0.94 & 4.2321 & 1.1033 & 66 & $15 / 1 / 2005$ \\
0.98 & 5.8513 & 1.0785 & 67 & $18 / 12 / 2005$ \\
0.98 & 23.963 & 1.4591 & 68 & $9 / 1 / 2005$ \\
\hline
\end{tabular}

acceptable degree. A general form of the linear model was selected to be of the form

$$
\mathrm{Y}=\mathrm{a} \cdot \mathrm{X}+\mathrm{b}
$$

where $\mathrm{X}$ is DN of a pixel in each band of LISS-III, $\mathrm{Y}$ is the calculated DN for the same band in $7 \mathrm{ETM}^{+}$. $a$ and $b$ are coefficients that can be determined through Least Square Method. The relation between observation and unknowns could be of the form;

$$
\mathrm{L}=\mathrm{A} \cdot \mathrm{X}
$$

where in this equation $\mathrm{L}$ is matrix of observation and $\mathrm{X}$ the matrix of unknowns and $\mathrm{A}$ is the matrix of coefficients. Then using least square method one can calculate Matrix of unknowns through the following equation:

$$
X=\left(\mathrm{A}^{\mathrm{T}} \mathrm{PA}\right)^{-1} \mathrm{~A}^{\mathrm{T}} \mathrm{PL}
$$

In Eq. 3, $\mathrm{T}$ stands for Matrix transpose and (-1) for inverse matrix. $P$ is the matrix of observation weights which can be considered as a unity matrix $(\mathrm{P}=\mathrm{I})$ if the observation precision were uniform.

After constructing model by determining $a$ and $b$ coefficients, for a given value of $\mathrm{X}$ from LISS-III, a value of $\mathrm{Y}$ for the same place in $7 \mathrm{ETM}^{+}$image will be determined. By applying Eq. 1 to red and NIR of LISS-III, two new matrices will be created with which we can fill up the missing places in $7 \mathrm{ETM}^{+}$images. The calculated coefficients of $\mathrm{a}$ and $\mathrm{b}$ in Eq. 1 is presented in Table 4.

At the end of this stage, the missing pixels in bands 3 and 4 of the $7 \mathrm{ETM}^{+}$will be fully recovered. In the next stage we will recover missing pixels of $7 \mathrm{ETM}^{+}$ images in other spectral bands.

Stage 2: Filling up empty spaces in bands 1, 2, 5, 6 and 7 of $7 \mathrm{ETM}^{+}$image Here we introduce two methods. 
Am. J. Applied Sci., 5 (6): 618-625, 2008

Table 5: The calculated coefficients of a and b in Eq. 4

\begin{tabular}{llrrc}
\hline $\mathrm{y}$ & $\mathrm{X}$ & $\mathrm{a}$ & $\mathrm{b}$ & Correlation coeff. \\
\hline 1 & 3 & 0.7687 & 13.2870 & 0.9260 \\
1 & 4 & 0.5160 & 14.4010 & 0.7167 \\
2 & 3 & 0.7564 & 6.8032 & 0.9757 \\
2 & 4 & 1.1254 & 20.1480 & 0.7566 \\
5 & 3 & 0.4201 & 24.2170 & 0.4721 \\
5 & 4 & 0.4549 & 13.1140 & 0.5895 \\
61 & 3 & -0.1271 & 116.2600 & 0.7906 \\
61 & 4 & -0.1116 & 117.8200 & 0.7094 \\
62 & 3 & -0.2351 & 122.8300 & 0.7828 \\
62 & 4 & -0.2091 & 125.9300 & 0.7117 \\
7 & 3 & 0.3659 & 15.4310 & 0.5181 \\
7 & 4 & 0.3363 & 9.8159 & 0.5400 \\
\hline
\end{tabular}

Method 1: As we introduced in the previous stage, by constructing a linear relationship between red and NIR bands of the products of LISS-III and $7 \mathrm{ETM}^{+}$sensor we could fill up the missing places in $7 \mathrm{ETM}^{+}$images. Since LISS-III does not have bands similar to other $7 \mathrm{ETM}^{+}$ bands, we should find some other methods for the replacement of the missing pixels in the other bands.

At this stage if we can find a rational relationship between recovered bands of $7 \mathrm{ETM}^{+}$(3 and 4) with other band of this sensor, then we might be able to recover $7 \mathrm{ETM}^{+}$images in other spectral bands. For this, about 140 pixels from variety of land cover classes all around the scene (except missing places) were sampled and their corresponding DN values were extracted.

Here again we assumed a linear relationship between bands 3 and 4 with other bands (which might not be true for all bands and all the time) and by using the same procedure as in stage 1 but with new $7 \mathrm{ETM}^{+}$ images in band 3 and 4 (instead of LISS-III images), we tried to recover the defected area of $7 \mathrm{ETM}^{+}$images in the rest of spectral bands. Therefore the equation will be of the form;

$$
\operatorname{ETM}_{y}^{+}=a \cdot \operatorname{ETM}^{+}+b
$$

where $\mathrm{ETM}^{+}$represents new DN value for the missing pixels in bands $y=1,2,5,6$ and $7, \mathrm{ETM}^{+}$is DN in bands 3 and $4(x=3,4)$. a and $b$ are coefficients and are shown in Table 5.

According to Table 5, correlation between band 3 with bands 1 and 2 is higher than 90\% (Fig. 3 and 4) so, recovering images in band 1 and 2 with the help of recovered band 3 is feasible. Low correlations between band 4 with bands 1 and 2 compared with band 3 could be due to the sudden change in spectral behavior of the vegetation in band 4. Also due to the spectral behavior of the soil and vegetation in bands 5 and 7 of $7 \mathrm{ETM}^{+}$,

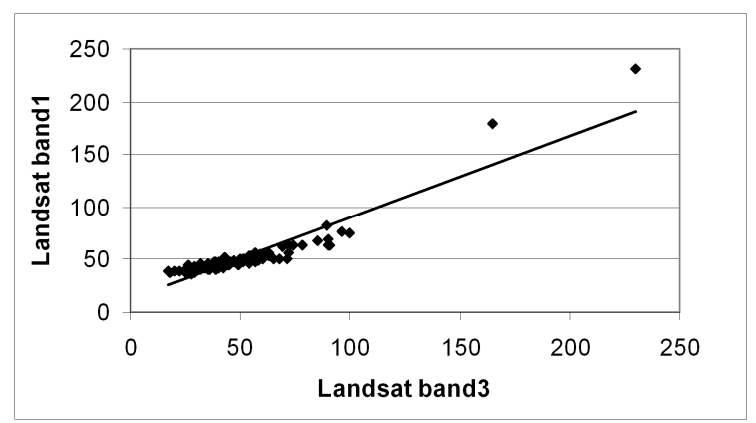

Fig. 3: Correlation between bands 3 and 1

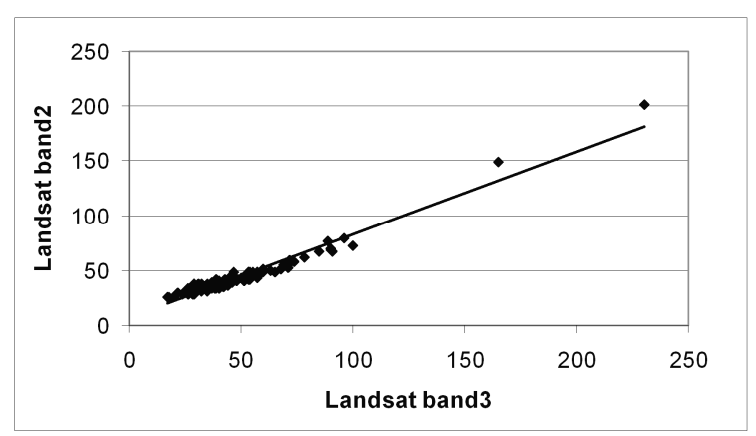

Fig. 4: Correlation between bands 3 and 2

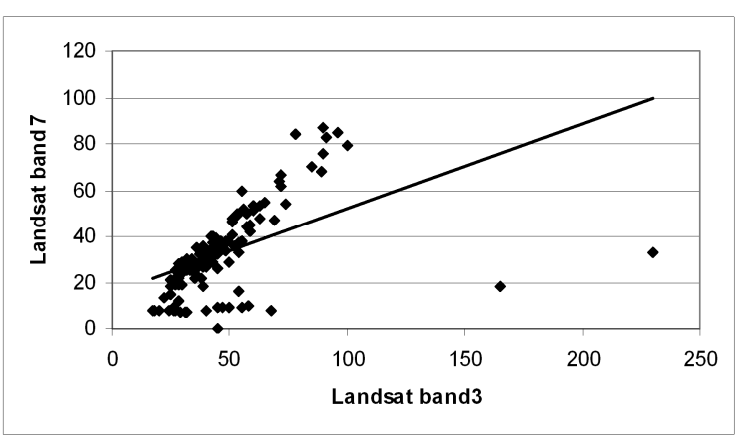

Fig. 5: Correlation between bands 3 and 7, while snow covered pixels are present

their correlations with bands 3 and 4 is lower in comparison with the correlation of bands 3 and 4 with bands 1 and 2 .

Note that in the shortwave IR region, snow has a special spectral behavior that is usually used to differentiate it from other surface features (Mobasheri, 2007). This might be the reason for low correlation between bands 3 and 4 with 5 and 6 . So, we masked snow pixels and we found that the correlation between bands 3 and 7 increased from 0.5181 (Fig. 5) to 0.7856 (Fig. 6). Higher correlation might be achieved if we 


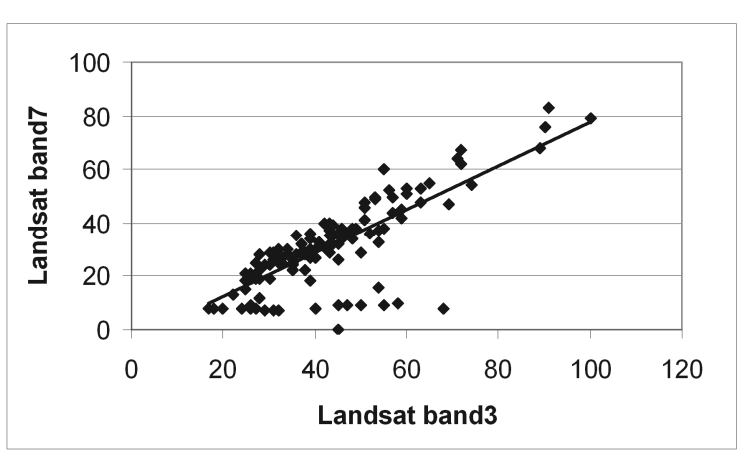

Fig. 6: Correlation between bands 3 and 7, with the absence of snow covered pixels

Table 6: Coefficients of Eq. 5

\begin{tabular}{lccl}
\hline $\mathrm{c}$ & $\mathrm{b}$ & $\mathrm{A}$ & $\mathrm{Z}$ \\
\hline 12.9529 & 0.4165 & 0.0588 & 5 \\
116.970 & -0.0242 & -0.1063 & $1-6$ \\
124.417 & -0.0541 & -0.1887 & $2-6$ \\
9.3490 & 0.2294 & 0.1708 & 7 \\
\hline
\end{tabular}

could resolve the content of each pixel through unmixing process.

With regard to the findings so far, if the land cover map be available then, linear model for different bands in each class cover could be executed but, usually this is not the case i.e. the land cover map is not always available and preparing such a map is time consuming so, we decided to use some other approaches for reconstruction of bands 5, 6 and 7 of $7 \mathrm{ETM}^{+}$.

Method 2: Our investigation showed that simultaneous use of bands 3 and 4 (which were reconstructed in the previous stage) and fitting a combined model to bands 5,6 and 7 (as a layer or surface) gives a much better correlation. Equation 5 may represent such a relation;

$$
Z=a \cdot X+b \cdot Y+c
$$

where $\mathrm{X}$ and $\mathrm{Y}$ are $\mathrm{DN}$ values of a pixel in band 3 and 4 , and $\mathrm{Z}$ is $\mathrm{DN}$ value of the corresponding pixel in bands 5,6 or 7. a, b and c are coefficients which are calculated and are shown in Table 6.

To calculate the correlation between $\mathrm{Z}$ with $\mathrm{X}$ and $\mathrm{Y}$, we used the following equation

$$
R_{x y z}=\frac{r_{x y}-r_{x z} \cdot r_{y z}}{\sqrt{\left(1-r_{x z}^{2}\right)\left(1-r_{y z}^{2}\right)}}
$$

Where $r_{i j}$ is correlation between two component of $i$ and $\mathrm{j}(\mathrm{i}, \mathrm{j}=\mathrm{X}, \mathrm{Y}, \mathrm{Z}$ and $\mathrm{i} \neq \mathrm{j})$ and is calculated from Eq. 7:

$$
\mathrm{r}_{\mathrm{ij}}=\frac{\mathrm{N} \sum \mathrm{ij}-\sum \mathrm{i} \sum \mathrm{j}}{\sqrt{\left[\mathrm{N} \sum \mathrm{i}^{2}-\left(\sum \mathrm{i}\right)^{2}\right] \times\left[\mathrm{N} \sum \mathrm{j}^{2}-\left(\sum \mathrm{i}\right)^{2}\right]}}
$$

Using Eq. 6 and 7, all different correlation coefficients were calculated and can be found in Table 7 .

\section{RESULTS AND DISCUSSION}

As we mentioned in previous sections, the objective of this work was to compensate for the malfunctioning of SLC in the $7 \mathrm{ETM}^{+}$sensor apparatus using IRS/1D LISS-III sensor products. Figure 7a is a color composite of 433 of $7 \mathrm{ETM}^{+}$before any corrections or modifications take place where a subset of the image is enlarged. Figure $7 \mathrm{~b}$ is the same image after amendment through the procedures mentioned in the previous sections.

In Fig. 8 and 9 images from bands 2 and 5 of the $7 \mathrm{ETM}^{+}$after corrections for SLC malfunctioning are

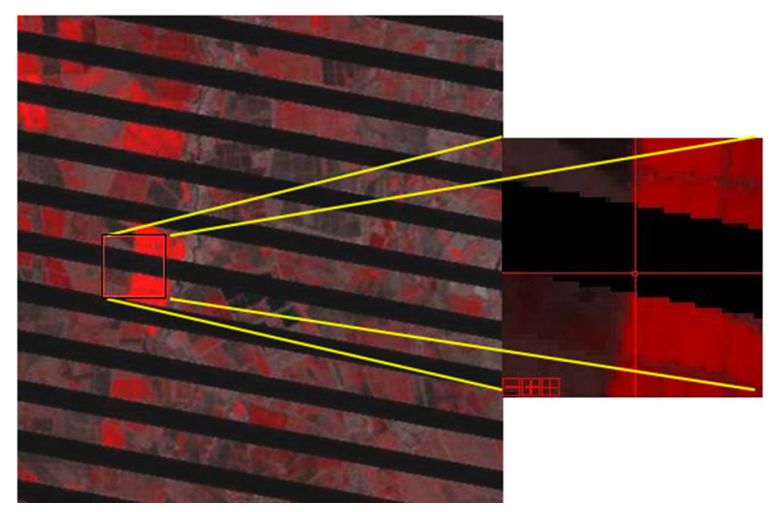

Fig. 7a: A 433 color composite of $\mathrm{ETM}^{+}$image before correction

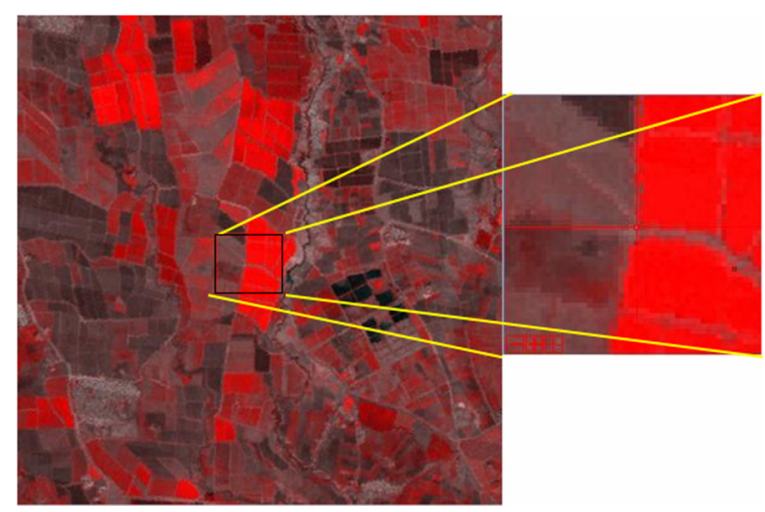

Fig. 7b: A 433 color composite of $\mathrm{ETM}^{+}$image after correction 
Table 7: Calculated correlation coefficients using Eq. 6 and 7

\begin{tabular}{lrrrl}
\hline $\mathrm{r}_{34 \mathrm{z}}$ & $\mathrm{r}_{4 \mathrm{z}}$ & $\mathrm{r}_{3 \mathrm{z}}$ & $\mathrm{r}_{34}$ & $\mathrm{Z}$ \\
\hline 0.7613 & 0.5176 & 0.3494 & 0.7913 & 5 \\
0.7546 & -0.3693 & -0.5164 & 0.7913 & $1-6$ \\
0.7542 & -0.3691 & -0.5084 & 0.7913 & $2-6$ \\
0.7448 & 0.4943 & 0.4006 & 0.7913 & 7 \\
\hline
\end{tabular}

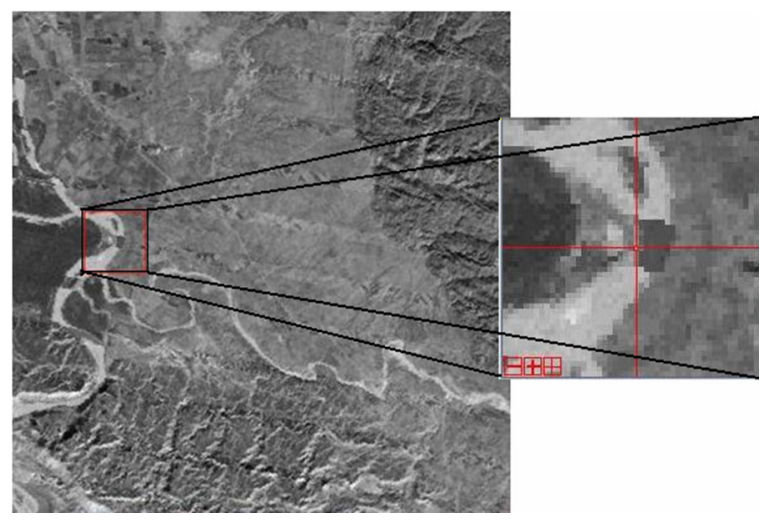

Fig. 8: Band 2 of 7ETM+ after correction for SLC defection

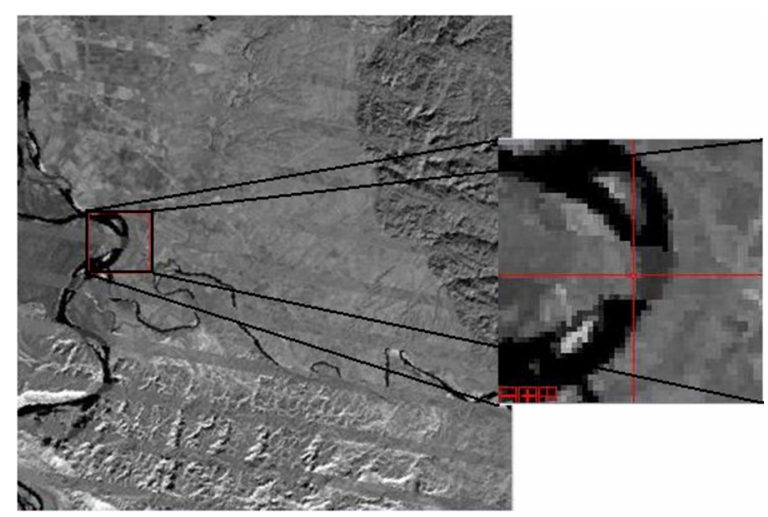

Fig. 9: Band 5 of 7ETM+ after correction for SLC defection

shown. However, since at this stage the reconstructed bands 3 and 4 have been used to modify the other bands of 7ETM, as expected, the precision of the results has decreased (Table 7). Changes in the environmental conditions and specially atmospheric optical depth and turbidity due to the differences in imaging time and their effects on the Landsat and IRS images, can affect the modification model severely (Fig. 8 and 9). As can be seen in Fig. 8, in the river portion of subset, the anomaly of the modification due to change in the environmental conditions can be seen, while in the edge of the river, reconstruction performs better.
Figure 9 show the corrected image of band 5 where in the river portion of the subset, the anomaly due to the modification is a result of changes in the environmental conditions while in the edge of the river, reconstruction shows better performances.

\section{CONCLUSION}

Regarding the unique specification of Landsat 7 satellite sensor such as its spectral bands in the shortwave IR and TIR region and due to the suitable spatial resolution which is ideal for most of the scientific researches, a technique for the reconstruction of the defected $7 \mathrm{ETM}^{+}$images was built up.

Due to the availability of IRS/1D LISS-III images for our region of interest i.e. southwest of Iran, it was decided to use these images to recover $7 \mathrm{ETM}^{+}$ products. Other images such as SPOT products also could be used equally.

The procedure of reconstruction of defected $7 \mathrm{ETM}^{+}$images is divided into two stages. In the first stage, after implementation of some preprocessing to both LISS-III and 7ETM ${ }^{+}$imageries and image to image registration, a linear model between bands 3 and 4 of $7 \mathrm{ETM}^{+}$and bands 2 and 3 of LISS-III was setup. This model enabled us to fill up the missing places in $7 \mathrm{ETM}^{+}$defected image and produce two new images in bands 3 and 4 .

In the second stage, we used these two newly reconstructed images of $7 \mathrm{ETM}^{+}$to recover images of $7 \mathrm{ETM}^{+}$in other spectral bands. At this stage we introduced two methods one using linear relation between band 3 and bands 1 and 2 and in the second method we introduced a planar relationship between bands 3 and 4 with each one of bands 5, 6 and 7. The models have applied to the whole scene and found to be fairly acceptable. During this study it is found that the following precautions must be taken into account when applying these models and methods. i- spatial, Radiometric, Temporal and Spectral resolutions of two sensors must be close enough to each other, ii- the images should be corrected relative to each other to an acceptable level, iii- the selected samples for the purpose of calculation of model coefficient should have suitable distribution among different surface features and iv-surface wetness should be preferably similar for all images i.e., meteorological parameters should be taken in to account.

\section{REFERENCES}

1. Landsat 7 Science Data Users Handbook, http://ltpwww.gsfc.nasa.gov/IAS/handbook/handbo ok_toc.html, NASA Godd ard Space Flight Center Landsat Project Science Office, Greenbelt, Maryland, August 2003. 
2. Doorn, B., 2003. SLC Anomaly Investigation. Report to US Dept. of Agriculture, Foreign Agriculture Service.

3. Landsat 7 Science Team, 2003. Preliminary Assessment of the Value of Landsat-7 ETM $^{+}$Data Following Scan Line Corrector Malfunctioning. US. Geological Survey, EROS Data Center, Sioux Falls, SD 57198.

4. Masek, J.G., 2003. Landsat 7 Anomaly Investigation. Report GSFC Code 923 to NASA.

5. Mobasheri, M.R., 2007. Fundamental of Physics in Remote Sensing and Satellite Technology. (Farsi Ed.). K.N. Toosi University of Technology Press, pp: 205-287.
6. Skole, D.L., J. Qi and J. Samek, 2003. Analysis of the Scan Line Corrector Anomaly Onboard Landsat 7 With Respect to Measurement and Mapping of Land Cover Change. Report of Michigan State University to NASA.

7. Vogelmann, J.E., D. Ohlen, B. Reed and S. Howard, 2003. Preliminary Assessment of the Value of Landsat $7 \mathrm{ETM}^{+}$Data Following Scan Line Corrector Malfunctioning: Views from the Sciences. Report SAIC, USGS/EROS Data Center.

8. WAN, Z., 1999. MODIS Land-Surface Temperature Algorithm Theoretical Basis Document (LST ATBD), Version 3.3, Institute for Computational Earth System Science, University of California, Santa Barbara. 